\title{
Cemented Constrained Total Hip Arthroplasty in a Patient With Osteogenesis Imperfecta and Recurrent Grand Mal Seizure Activity
}

\author{
Sharon Kermecia, c, d, Patrick Weinraucha, b, c
}

\begin{abstract}
We present the management of secondary osteoarthritis due to posttraumatic femoral head osteonecrosis in a patient with osteogenesis imperfecta and poorly controlled grand mal seizure activity using cemented constrained total hip arthroplasty devices. The use of a cemented constrained acetabular implant has provided a successful outcome in this high-risk presentation.
\end{abstract}

Keywords: Osteogenesis imperfecta; Constraint; Cement; Arthroplasty; Hip; Freedom; Biomet

\section{Introduction}

Osteogenesis imperfecta (OI) is a genetic disorder effecting the production of type 1 collagen with clinical manifestations relating to altered bone and connective tissue structure [1]. Orthopedic manifestations of OI include high fracture rates and variable degrees of joint laxity, muscular weakness and spinal scoliosis, depending on severity of the condition [2]. Type 1 OI accounts for $50-60 \%$ of all people diagnosed with the condition [3].

With improving life expectancy of people with OI, increasing numbers of patients with this condition are becoming candidates for joint replacement surgery. Total hip arthroplasty (THA) in patients with OI may be associated with an increased risk of prosthetic instability and peri-prosthetic fractures due to bone fragility and ligamentous laxity [4]. As a result, the long-term outcomes from primary THA in patients with OI are potentially jeopardized due to increased risks of arthroplastyrelated complications.

Manuscript accepted for publication December 18, 2015

aBrisbane Hip Clinic, St Andrews War Memorial Hospital, Queensland, Australia

${ }^{b}$ School of Medicine, Griffith University, Queensland, Australia

'St Andrews War Memorial Hospital, Queensland, Australia

${ }^{\mathrm{d}}$ Corresponding Author: Sharon Kermeci, Brisbane Hip Clinic, Level 7, St Andrews War Memorial Hospital, 457 Wickham Terrace, Spring Hill Q 4000, Australia. Email: reception@brisbanehipclinic.com.au

doi: http://dx.doi.org/10.14740/jmc2398w
Patients suffering epilepsy, particularly in the presence of grand mal seizure activity, are at higher risk of musculoskeletal injury and complications after joint arthroplasty, especially if when co-existent risk factors for fragility fractures such as OI are present. Seizure activity increases the danger of proximal femoral and acetabular fractures, as well as prosthetic hip instability and dislocation [5-7].

Concern associated with the use of constrained liners relates to the potential for higher rates of aseptic loosing secondary to early prosthetic impingement and reduced functional range of motion. Contemporary data from the Australian National Joint Replacement Registry [8] however demonstrate favorable revision rates at 10 years with the use of constrained devices even in high-risk populations.

We describe the management of secondary post-traumatic hip osteoarthritis in a high-risk patient with a combination of both OI and poorly controlled grand mal seizure activity. We discuss the use of cemented implants for the treatment of osteoarthritis in patients with OI and the use of a cemented constrained device in this unique presentation.

\section{Case Report}

A 46-year-old woman with type 1 OI presented with osteoarthritis secondary to femoral head osteonecrosis as a result of a displaced subcapital femoral fracture previously managed by internal fixation (Fig. 1). Past medical history included poorly controlled grand mal seizure activity, resulting in numerous fractures in the context of her skeletal fragility.

On the basis of significant hip articular symptoms and functional disability, definitive treatment by THA was recommended. Risk factors considered for this patient included the high risk of dislocation due to seizure activity and also the increased risk of peri-prosthetic fractures due to bone fragility. Pre-operatively we considered the use of a constrained arthroplasty device as favorable due to the presence of recurrent seizure activity; however, we remained concerned about the use of uncemented devices due to the risk of intra-operative fracture during implant impaction. In particular, this concern related to the risk of pelvic fracture during implantation of an uncemented constrained acetabular prosthesis.

THA was performed via a posterior approach using a cemented Freedom acetabular implant (Biomet Orthopaedics, 


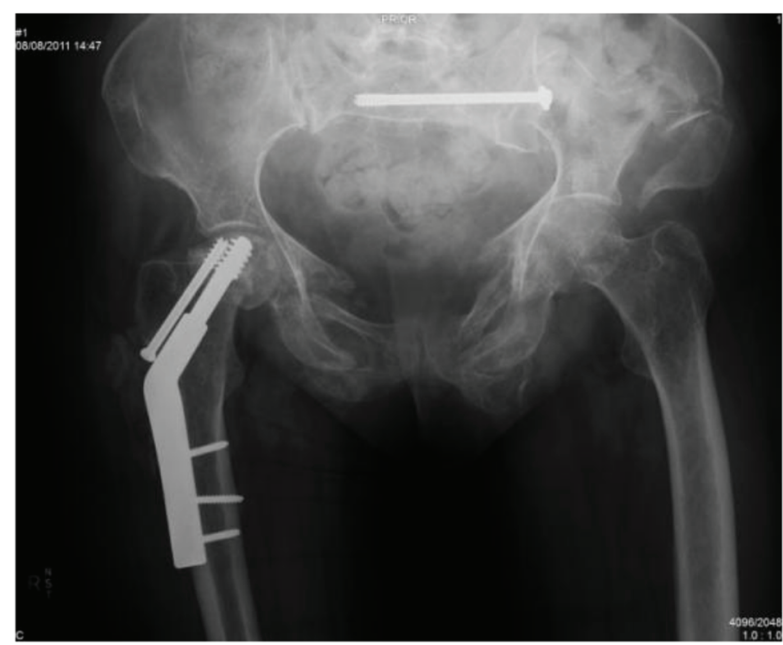

Figure 1. Femoral head collapse and secondary osteoarthritis due to post-traumatic osteonecrosis.

Indiana, USA) and a cemented double tapered polished femoral stem. For implantation of the femoral component, no cement restrictor was used, allowing the cement to pass distally into the medullary canal for additional bone reinforcement below the femoral component to reduce the risk associated with a distal stress riser at the tip of the femoral component.

Post-operative radiographs are demonstrated in Figure 2. The patient was permitted to weight-bear as tolerated post-operatively and the immediate pre-operative recovery progressed uneventfully.

At both 6-week and 6-month post-operative reviews, the patient was progressing well with substantial improvement in pain and functional capability. Two years following the right total hip replacement, as a result of a fall unrelated to the index procedure, the patient sustained a left (contralateral) proximal femoral fracture. This was managed by internal fixation us-



Figure 2. Immediate post-operative radiograph demonstrating a fully cemented constrained total hip arthroplasty. Note the absence of a femoral cement restrictor with passage of cement distal to the femoral stem.



Figure 3. Three-year post-operative radiograph. Patient suffered a left hip fracture in the year prior to the radiograph being taken. Satisfactory clinical and radiographic outcomes obtained from the right total hip arthroplasty.

ing an intramedullary nail. The right hip replacement remained well functioning.

At most recent review, approaching 4 years post-arthroplasty, the patient presents a well functioning prosthetic hip joint with successful clinical and radiologic outcomes (Fig. 3 ). Although the patient experiences difficulties in performing functional activities due to other musculoskeletal conditions associated with her OI, the right hip joint is pain-free and she remains highly satisfied with the result.

\section{Discussion}

Prosthetic instability remains a leading cause for revision of THA in modern practice [9-11]. Patients at risk of prosthetic hip instability include those with neuromuscular disorders such as epilepsy, particularly when presenting with grand mal seizure activity. When considering the appropriate prosthetic selection for this patient group, consideration must be made to the increased risk of both peri-prosthetic fracture and dislocation.

For the treatment of this complex presentation of combined skeletal fragility and instability risk, limited literature is available to guide appropriate arthroplasty management. Cemented implants were chosen due to concerns with bone fragility and the suitability of press fit implants due to the risk of intraoperative peri-prosthetic fracture during impaction and the increased risk of post-operative fractures, particularly about the femoral component. Another potential advantage of using a cemented femoral component relates to the ability to reinforce the distal femur with cement, reducing the potential for fracture creation at the tip of the femoral implant due to stress transition. To address concerns regarding dislocation and instability, especially when considering the patient's seizure activity, a constrained acetabular implant was chosen. Hernigou et al [12] support the use of constrained acetabular implants in patients with neurological or cognitive impairment, demonstrating a $2 \%$ failure 
rate in constrained devices compared to an $18 \%$ failure rate in unconstrained devices at 5 years.

The use of constrained devices, particularly in younger patients, is less common in modern arthroplasty practice. This is due to the perception of complications of complications relating to the use of constraint including loss of fixation, early aseptic loosening, liner disassociation, and material failure [13]. As most constrained acetabular components currently used are modular uncemented devices, the impaction of an uncemented acetabular component in our patient also represented increased threat of acetabular or pelvic fracture. Consequently, the decision to use a cemented acetabular component was made to address this concern. When using constrained total hip implants, to address the potential for early prosthetic impingement and associated loss of functional range of motion, we favored the use of the Freedom implant (Biomet Orthopaedics, Indiana, USA). The Freedom constrained implant allows secure prosthetic constraint whilst providing an enhanced range of motion prior to impingement of up to $114^{\circ}$ (in $36 \mathrm{~mm}$ head devices) and offers both cemented and uncemented variants [14]. Particularly in comparison to other constrained devices, Freedom constrained acetabular implants have demonstrated highly favorable results $[15,16]$.

\section{Conclusion}

In summary, we describe the successful conduct of a fully cemented and constrained THA in a patient at high risk of periprosthetic fractures and prosthetic instability. In particular, we have found the use of a cemented constrained acetabular device useful in this unique clinical presentation.

\section{Conflict of Interest}

The authors declare no conflict of interest related to the content of this manuscript.

\section{References}

1. Sillence DO, Senn A, Danks DM. Genetic heterogeneity in osteogenesis imperfecta. J Med Genet. 1979;16(2):101116.

2. Liporace FA, Yoon RS, Frank MA, Maurer JP, Gaines RJ. Single-stage total hip arthroplasty and fracture fixation for a both column acetabular fracture in type I osteogenesis imperfecta. Injury. 2011;42(10):1184-1187.

3. Foundation OI. OI Issues: Type 1 - Understanding the Mildest Form of Osteogenesis Imperfecta. 2015; http:// www.oif.org. Accessed 26/07/2015, 2015.

4. Papagelopoulos PJ, Morrey BF. Hip and knee replacement in osteogenesis imperfecta. J Bone Joint Surg Am. 1993;75(4):572-580.

5. Atilla B, Caglar O, Akgun RC. Acute fracture of the acetabulum secondary to a convulsive seizure 3 years after total hip arthroplasty. Orthopedics. 2008;31(3):283.

6. Murzic WJ, Taylor JK, Bargar WL. Seizure-induced femur fracture after total hip replacement. Orthopedics. 1993;16(8):906-908; discussion 908-909.

7. Grimaldi M, Vouaillat H, Tonetti J, Merloz P. Simultaneous bilateral femoral neck fractures secondary to epileptic seizures: treatment by bilateral total hip arthroplasty. Orthop Traumatol Surg Res. 2009;95(7):555-557.

8. Association AO. National Joint Replacement Registry. Annual Report. Adelaide: AOA; 2015.

9. Registry NZJ. The New Zealand Joint Registry Fourteen Year Report - January 1999 to December 2012. Wellington: NZOA; 2013.

10. Association AO. National Joint Replacement Registry. Annual Report. Adelaide: AOA; 2014.

11. Registry NJ. National Joint Registry for England, Wales and Northern Ireland 11th Annual Report. Hertfordshire: NJR; 2014.

12. Hernigou P, Filippini $\mathrm{P}$, Flouzat-Lachaniette $\mathrm{CH}$, Batista $\mathrm{SU}$, Poignard A. Constrained liner in neurologic or cognitively impaired patients undergoing primary THA. Clin Orthop Relat Res. 2010;468(12):3255-3262.

13. Yun AG, Padgett D, Pellicci P, Dorr LD. Constrained acetabular liners: mechanisms of failure. J Arthroplasty. 2005;20(4):536-541.

14. Lombardi AV, Jr. Constrained liners in revision: total hip arthroplasty an overuse syndrome: in opposition. J Arthroplasty. 2006;21(4 Suppl 1):126-130.

15. Berend KR, Lombardi AV, Jr., Mallory TH, Adams JB, Russell JH, Groseth KL. The long-term outcome of 755 consecutive constrained acetabular components in total hip arthroplasty examining the successes and failures. J Arthroplasty. 2005;20(7 Suppl 3):93-102.

16. Berend KR, Lombardi AV, Jr., Welch M, Adams JB. A constrained device with increased range of motion prevents early dislocation. Clin Orthop Relat Res. 2006;447:7075. 